\title{
FORMACIÓN DE UN ESPÍRITU CIENTÍFICO EN EDUCACIÓN BÁSICA DESDE LA ENSEÑANZA DE LAS CIENCIAS NATURALES
}

\author{
Por: Zulman Estela Muñoz Burbano ${ }^{1}$ - Sandra Yaneth Cerón Cabrera ${ }^{2}$
}

\section{RESUMEN}

La actualidad abre un espacio para la reflexión en torno a la enseñanza de las Ciencias Naturales; situación evidente en el análisis de las prácticas pedagógicas que se centran en contenidos y no en procesos. Al respecto, se propone considerar al educador, más que al discente, como punto de partida de un proceso, que permita pasar del énfasis de las gruesas informaciones descontextualizadas a la dinámica del diálogo de saberes, y también a un acercamiento de los estudiantes al conocimiento de la naturaleza de las ciencias y al desarrollo de un espíritu científico, que vaya más allá de simples acciones de indagación o consulta. La formación y la adquisición de un espíritu científico entendido como una estructura compleja en la que se articula el pensamiento crítico, destrezas relacionadas con la observación y la capacidad de confrontación y argumentación de ideas entre otros, están ligadas con la didáctica del docente, con la forma como asume su quehacer y cómo él se relaciona con la ciencia y la actividad científica.

Palabras Clave: enseñanza de las ciencias, espíritu científico, práctica pedagógica.

Clasificación JEL: 121.

1. Magister en Educación, Especialista en Docencia de la Química - Especialista en Docencia Universitaria - Docente Universidad de Nariño. Investigadora.Grupo GIDEP. zulmamu0706@hotmail. com

2. Magister en docencia Universidad de la Salle - Especialista en Docencia Universitaria - Licenciada en Español y literatura - Instituto Champagnat Pasto. sandraceron27@gmail.com 


\title{
FORMING A SCIENTIFIC SPIR IT IN BASIC EDUCATION FROM THE TEACHING OF NATURAL SCIENCES.
}

\author{
By: Zulman Estela Muñoz Burbano - Sandra Yaneth Cerón Cabrera
}

\begin{abstract}
The present reality opens up a space for reflection about the teaching of Natural Sciences which is an evident situation in the analysis of teaching practices that focus on content and not on processes. In this regard, it is proposed to consider the educator, rather than the learner, as a starting point for a process that allows to move from the emphasis on the heavy decontextualized information to the dynamics of dialogue of knowledge, and also to a rapprochement of the students to the understanding of the nature of the sciences and to the development of a scientific spirit which goes beyond simple acts of inquiry or consultation. The formation and acquisition of a scientific spirit understood as a complex structure in which critical thinking, observation-related abilities and the capacity to confront and support ideas, among others, is articulated with the teacher's didactics, the way in which they assume their work, and the way how they relate to the science and the scientific activity.
\end{abstract}

Key words: teaching of sciences, scientific spirit, and pedagogical practicum. JEL Classification: 121. 


\section{Introducción}

En el medio educativo la enseñanza y el aprendizaje de las Ciencias Naturales se ha convertido en el centro de innumerables reflexiones, y hoy son importantes objetos de estudio tanto en Colombia como en América Latina. Todo, quizá por la dificultad que se ha presentado en la formación y desarrollo de un espíritu científico por parte de los estudiantes. Este artículo se presenta como alternativa de reflexión en torno al docente y su práctica, a fin de lograr pasar de la adquisición de contenidos a conocer la naturaleza de la ciencia y a la comprensión de los fenómenos naturales.

El desarrollo de una concepción y práctica pedagógica tradicional es un hecho latente en la escuela colombiana; evidencias que apoyan esta idea se observan en los salones de clase, donde en muchas ocasiones, se implementan acciones encaminadas hacia la asimilación de contenidos e informaciones poco significativas, situación seguida de una escasez de comprensión e interpretación de la naturaleza de la ciencia y de poco fortalecimiento de actitudes científicas, valores y habilidades de pensamiento que proyectan la formación de un espíritu científico en los educandos.

En la mayoría de las instituciones educativas colombianas, la enseñanza de las Ciencias Naturales se ha caracterizado por su énfasis en los contenidos, los cuales están presentes en diversos documentos de editoriales, sin que se haya dado la posibilidad de detenerse en procesos tales como el significado correcto de los referentes y el argumento formal que los justifica (Porlan, 1993). Vale decir, que en muchos casos, los conceptos han ocupado el lugar relevante del trabajo en ciencias, sin crear espacios de reflexión para que el estudiante conozca el origen de dichas categorías conceptuales, y el proceso que permite su construcción; entonces se convierte a quien aprende en receptor de datos; Freire afirma: La narración los transforma en "vasijas", en recipientes que deben ser "llenados por el educador", cuanto más se van llenando los recipientes con sus depósitos, tanto mejor educador será. Cuanto más se dejen dócilmente "llenar", tanto mejores educandos serán" (1996: 78). Al respecto es relevante mencionar que habilidades de pensamiento como el análisis, síntesis, inferencia, observación y resolución de problemas se han dejado de lado por realizar ejercicios de memoria, los cuales son base para la evaluación, en el aula de clase.

Las Ciencias Naturales se enseñan desde un modelo que no está acorde con las demandas de la sociedad postmoderna (Locarnini, 2007). La tendencia "bancaria" predomina, en muchos docentes de Ciencias Naturales de Colombia, quienes no han asumido el papel como facilitadores de un proceso; más aún, no tienen confianza ni visión a la hora de abordar su labor; muy por el contrario el objetivo que alivianaría esta situación debería tener como centro la formación del espíritu científico en los estudiantes, mediante el desarrollo de habilidades de pensamiento. Se sabe que con una adecuada motivación e integración de prácticas y saberes se podría ayudar a otros a descubrir el potencial que existe en ellos para que se conviertan en constructores del conocimiento. Se reconoce además, que a partir de esa condición creativa que caracteriza a los niños, las niñas y los adolescentes se pueden dinamizar acciones que reten el pensamiento, que permitan solucionar 
problemas y hacer proyecciones hacia un futuro emprendedor. Con el panorama antes expuesto, es preciso sentar bases en la realidad y mencionar que existen carencias presentes en el personal docente: en principio, se deben a los procesos de formación que ellos y ellas recibieron; dicha educación está basada en el memorismo acompañado de la rigidez didáctica.

En la práctica pedagógica de los docentes, siempre subyace un referente teórico pedagógico y epistemológico en el que suscribe su quehacer (Aduriz: 2001), sin embargo, se presenta un problema en cuanto a que el educador no siempre es consciente de este referente, o no tiene claro dentro de qué teoría o corriente está inserto -tampoco se ha preocupado por ello-. El referente teórico que posee el docente es básico en la forma de asumir el proceso de enseñanza, y de alguna manera, también es clave para el sentido que le imprima al aprendizaje de los conceptos y los distintos procesos. Es por eso que para la enseñanza de las Ciencias Naturales, la construcción de un concepto epistemológicamente válido permitirá comprender su naturaleza, su dinámica y el papel que juega la ciencia para la humanidad.

\section{De la importancia del cambio didáctico}

En el proceso de relación y apropiación del conocimiento científico que se desarrolla en las instituciones educativas, la mayoría de las veces, los docentes recurren a prácticas pedagógicas facilitadoras, atractivas e interesantes -con el firme propósito de lograr que los estudiantes aprendan-, pero con las que desafortunadamente ocurre el efecto contrario, porque son paralizantes (Aguirre: 2006); se debe agregar que los problemas se solucionan de forma superficial, no obstante, las acciones pedagógicas resultan castrantes; aclarando: son efectivas soluciones para los modelos tradicionales de evaluación, sin embargo, intrascendentes para el pensamiento; atractivas pero triviales; que no contribuyen a un aprendizaje científico.

De acuerdo con Hernández (2004), existe desconocimiento por parte de los educadores de las formas y estilos de aprendizaje de los estudiantes. Es así como se reconoce que los docentes no cuentan con los suficientes elementos para crear ambientes y desarrollar experiencias en el aprender a aprender. La situación anterior es una causa para que la memorización le gane al fortalecimiento de diversas habilidades de pensamiento: situación pedagógica que tiene como eje central el reconocimiento del estudiante como un sistema cambiante. Las estructuras cognitivas humanas tienen la posibilidad de modificarse de acuerdo con los estímulos que le proporcione el ambiente. Así, todo parte del tipo de experiencia del organismo, la cual puede ser propia o mediada. De acuerdo con Piaget (1966), De Bono (1986), Feuerstein (1993), Sternberg y Spear-Swerling (2000), todo proceso cognitivo que se desarrolle desde la infancia, se dinamiza en la adolescencia y se fortalece en edad adulta, además, las habilidades de pensamiento que se desplieguen en la edad escolar, ayudan a las personas a forjar mejores respuestas en la vida adulta. Teniendo en cuenta esta situación, la escuela está llamada a desarrollar estrategias para fomentar aprendizajes significativos en los estudiantes, pues hoy, no es suficiente tener información y conocimiento, es preciso saber utilizarlo.

Desde esa perspectiva, se trata de fomentar en las aulas una enseñanza que sea verdaderamente democratizadora, mediante la implementación de un estilo 
pedagógico que problematice los saberes y que sea una puerta de entrada hacia la búsqueda de nuevas soluciones, es decir, que conlleve al cuestionamiento y a la crítica, para plantear nuevos problemas (Gil, 1994); lo anterior no puede considerarse sencillo, de hecho, es un proceso complejo que implica una transformación profunda en la manera de contemplar la práctica pedagógica en el aula, es difícil generar una actividad escolar científica, pero es prioritario lograr algo más que una simple alfabetización científica.

El cambio es importante si se quiere fomentar un espíritu científico. Una transformación donde la marca sea el aprender situaciones para la vida, donde el pensamiento sea la máquina que auto-motiva a la persona. Según Luria, El pensamiento es un acto dinámico integral... La palabra es el instrumento fundamental del pensamiento" (1984: 323). Por su parte, Mayer, explica que "El pensamiento es la manipulación de la información percibida, aprendida y recordada" (1986: 22). De su lado Vega menciona que El pensamiento implica una actividad global del sistema cognitivo, con intervención de los mecanismos de memoria, atención, las percepciones o procesos de comprensión; pero no es reductible a éstos (1989: 439). Como se puede observar, las tres definiciones se relacionan con la organización de información, lo cual es una acción que pasa de reconocer datos fríos y sin sentido a convertirse en situaciones de lectura crítica para construir conocimiento. El cerebro humano establece diversas redes cognitivas; el número de redes aumenta cuando el pensamiento trabaja con la Información y la estructura en conocimiento, se establece entonces, que las características de un espíritu científico se vuelven herramientas para continuar aprendiendo de manera significativa.

\section{De la importancia de la historia de la ciencia}

La historia de la ciencia es un referente fundamental para que los educadores inviten a un verdadero diálogo de saberes, en ese sentido, se establece que es importante en la enseñanza de las Ciencias Naturales, abordar la filosofía y la historia, puesto que llevaría a construir una concepción, que lejos de la mitificación y la singularidad, permita establecer una idea de ciencia más plural, dinámica, y por tanto, plausible; de esta manera se va a reconocer a la ciencia más que como un producto acabado, como el resultado de un proceso de construcción social.

La historia y la filosofía de las ciencias jugarán un papel importante, al configurarse como un espacio de reflexión en torno al estatuto de cientificidad; los objetos de estudio, los criterios de verdad o las características que comporta el trabajo científico al interior de las disciplinas son situaciones de pensamiento para que un historiador de las ciencias - llámese profesor- mientras viaja al pasado, ayuda a otros a tomar conciencia del valor de la ciencia en la actualidad (Bachelard: 1988). Asimismo, la historia de las ciencias podría generar una Ambientación de las ideas epistemológicas, además de que provee de una buena fuente de "episodios paradigmáticos" (Aduriz, 2005: 6).

En los procesos pedagógicos, en ningún caso, se puede hablar de una receta que permita solucionar problemas, pero para la situación específica que se está abordando, se presentan, a continuación, algunas reflexiones susceptibles de ser 
llevadas a la práctica, en las cuales la historia de las ciencias podría ser valiosa al asumirla de una manera epistemológicamente válida:

1. Es fundamental evitar dar una visión simplista de la evolución de las ideas científicas, como si las teorías aparecieran por arte de magia, sin un proceso de construcción y maduración.

2. Con frecuencia, las teorías científicas se presentan simplemente como verdaderas o falsas, sin su respectiva contextualización. De manera habitual son calificadas a la luz del conocimiento actual, sin tener en cuenta que en su momento fueron útiles en la interpretación de la realidad, pues eran parte del acervo de conocimientos con que se disponía. El presentar las teorías de esta manera, tal vez genera un impacto negativo, pues las ideas previas de los alumnos sobre determinados temas, pueden relacionarse con ese tipo de saberes y generar frustración al considerar irrelevantes o poco plausibles (Gallego y otros, 2009).

3. Tradicionalmente los docentes tienen como objetivo en la enseñanza de las Ciencias Naturales demostrar la importancia que en investigación tiene el método científico, pero se puede caer en el error de presentar que el progreso científico solo está basado en la aplicación categórica de un método específico. No se trata tampoco de descalificar este método algorítmico, porque pudiera ser utilizado como un recurso didáctico interesante, (Arduriz, 2001); es necesario mostrar las limitaciones del proceso, y a la vez, entrar a dialogar con los estudiantes sobre la importancia del azar, la casualidad, que han sido hechos fundamentales en la construcción de la ciencia -desde luego no azar o casualidad aisladas, sino bajo el análisis de mentes críticas y reflexivas, que no dejaron pasar por alto dichos acontecimientos-. Esto además llama mucho la atención de los niños y estudiantes, genera apego y gusto por las ciencias al evidenciar la cotidianidad en ella.

4. Es importante desmitificar a los científicos, su vida y obra mediante la narración de hechos anecdóticos y aspectos de su vida personal; trabajar además el espíritu ético de la construcción del conocimiento y de quienes lo construyen. Con estas descripciones se hace más interesante la ciencia, más cercana al estudiante llevando, lo más probable, a enamorarse y sentirse parte de ese constructo.

5. Se debe también, tener en cuenta las ideas que el educando tiene sobre los temas que se van a estudiar, pues son de suprema importancia para el anclaje de su nuevo aprendizaje, así lo recalca Freire (1996: 19), en su Pedagogía de la autonomía: Enseñar exige respeto por los saberes de los educandos... el deber no es solo respetar los saberes con los que llegan los educandos... sino también... discutir la razón de ser de esos saberes en relación 'con la enseñanza de esos contenidos'.

6. El jugar con las ideas previas de los estudiantes, proporcionarán varias posibilidades que enriquecerán la enseñanza de las Ciencias Naturales (Muñoz, 1996); en lugar de simplemente escucharlas, o lo más grave, ignorarlas, se entra con 
ellas en un proceso de falsación y refutación, el estudiante siente desequilibrio, pero si se convence de que es más plausible el nuevo conocimiento, no lo va a memorizar de forma impositiva, lo va a aprehender y comprender, porque entra a su estructura conceptual tras un desarrollo dinámico que implica el cambiar lo que sabe por un saber que se aplica y se estructura para dar mayor número de explicaciones asertivas a la realidad que lo rodea.

7. El aprendizaje de las ciencias se dinamiza con el desarrollo de habilidades de pensamiento: analizar, sintetizar, clasificar, solucionar problemas. Para potenciar el espíritu científico en los estudiantes se hacer necesario motivar la lectura crítica de las distintas situaciones presentes en laboratorios.

\section{De la nueva formación de los estudiantes}

Es necesario conceptualizar y reorientar aquello que se entiende por espíritu científico. Las nuevas formas de concebir el mundo, y con ellas, el surgimiento de nuevos problemas conllevan a asumir el espíritu científico como la esencia del cambio que se propone para lograr una enseñanza de las ciencias, que trascienda la acumulación obediente de contenidos. Se parte del criterio, ya enunciado, de considerar la relación de las ciencias en las instituciones educativas como un proceso de construcción que lleva a la apropiación del conocimiento y a la estructuración de un pensamiento y de unas actitudes de corte científico.

El espíritu científico se define como una actitud o disposición subjetiva a la búsqueda de soluciones (Ruiz, 2006: 100) que se puede suscribir en tres aspectos básicos:

1. capacidad para convertir situaciones particulares en problemas de investigación, es decir, capacidad para ver y formular problemas;

2. actitud o disposición del estudiante que busca métodos adecuados para encontrar soluciones al problema que pretende resolver;

3. desarrollo de una mente crítica y racional, dispuesta siempre a algo más de aquello que ocurre en el aula, capaz de trascender las paredes que encierran las acciones de apropiación del conocimiento, para buscar fuera de ellas soluciones, pero a la vez más problemas.

El espíritu científico se relaciona con un pensamiento crítico, y dentro de la escuela debería ser el fruto de un proceso que inicie desde el preescolar, desarrollando habilidades y destrezas del pensamiento tales como la observación, la contrastación, la clasificación, inducción, deducción, planteamiento de inferencias, la composición y la descomposición entre otras; resaltando la importancia de hacer conjeturas y sustentarlas. Además, el espíritu científico se fortalece con el desarrollo del buen juicio, con el discernimiento suficiente para estar en capacidad de separar lo simplemente casual de lo verdaderamente esencial, es decir, mentes con la suficiente formación para separar contenidos sin mucha relevancia de aquellos que son más significativos para la construcción de otros y para aplicarlos en los 
planos de resolución en situaciones problémicas. En otras palabras, el educando puede contar con diversas capacidades de reflexión que le ayuden a gestionar la información que circula a su alrededor, pero también usa lo que sabe para plantear soluciones creativas a los problemas y situaciones expuestas en su medio, se está, entonces, pasando de un pensamiento pasivo a otro crítico y creativo.

El docente será quien dé origen a escenarios donde se fortalezca el desarrollo de esas habilidades, también de la interacción con los demás, fomentando la autonomía y la creatividad; dichos escenarios pueden ser reales o virtuales, reales con la exploración del medio, del entorno inmediato, del mismo cuerpo; hipotéticos, con el planteamiento de situaciones que si bien son cotidianas se problematizan y se resuelven de forma teórica.

En el medio educativo se vincula al espíritu científico directamente con la investigación, entendida desde la aplicación del método científico, pero en la escuela, y en especial, en la educación básica se debería hablar de actividad científica escolar (Izquierdo y Aduriz, 2002), la cual estaría determinada por la formación de actitudes científicas; esto evitará un enfoque que lleve a una unidireccionalidad del pensamiento de los estudiantes que les coarta la libertad, la imaginación y la creatividad al no poder buscar diferentes caminos y personalizarlos al tratar de llegar a resolver una situación problema, cuando lo que verdaderamente debería buscarse es un diálogo entre la razón y el sujeto mismo (Touraine, 2000).

De igual manera no se puede desconocer que, además de las características descritas y en aras de una formación integral, el espíritu científico debe estar permeado por la ética, la humildad, la seriedad, el respeto por las ideas de los demás y por la verdad; de capacidad para superar obstáculos, sortear situaciones, enfrentarse al fracaso, pensar en el error como agente dinamizador, entre otros, sin olvidar la relevancia de la escritura y la lectura. ${ }^{3}$ En síntesis, la formación del nuevo espíritu científico de los estudiantes debe responder a una educación de carácter integral, en la que se presente unidad en la relación conocimiento-espíritu, superando así la dualidad que se desprende de la concepción cartesiana de "pienso luego existo".

Cuando a la práctica del docente se vincula a la historia de las ciencias se abre la posibilidad de la reflexión ética de construcción de conocimiento y los factores que influyen en el mismo, permitiendo además cultivar valores en los estudiantes tales como la confianza en sí mismo, el respeto por las ideas de los demás, el liderazgo, trabajo en equipo, la confrontación de sus ideas, el respeto por el entorno, llevando de esta manera a la formación del estudiante, no solo en conocimiento, sino en valores.

Los docentes colombianos deben asumir la responsabilidad de desarrollar en sus estudiantes actitudes científicas, que a su vez, desemboquen en la formación

3. En 1820, don Andrés Bello sentenció que "Las artes de leer y escribir son como los cimientos sobre los que descansa todo el edificio de la literatura y de las ciencias". Hoy, casi doscientos años después, ello resulta ser la más orientadora verdad. 
de un espíritu científico. En ese sentido, vale la pena reconocer que para que se cumpla dicho objetivo, el maestro debe mostrar actitudes de motivación para la enseñanza tales como:

Curiosidad. Despertar el deseo de conocer, de hacerse preguntas implica motivar en el niño y en el adolescente su capacidad de asombro, sobre todo en aquellos hechos que contradicen lo aprendido; tener gusto por el saber, por la manipulación, por la experimentación y ser curioso es un instinto de supervivencia y gracias a la evolución, el hombre posee esta ventaja natural para la construcción del conocimiento.

Creatividad. Considerar direcciones múltiples al implementar el pensamiento divergente, es ser capaz de encontrar la solución a un fenómeno nuevo, concebir un gran número de hipótesis o de soluciones originales y variadas a un problema dado.

Confianza en sí mismo. Encontrar solución por sí mismo, esto es buscar información en lugar de pedírsela al profesor. Que el estudiante crea en lo que sabe y aplique ese conocimiento.

Pensamiento crítico. Estar dispuesto a basarse en la experiencia para volver a dudar de las representaciones personales, así como de las afirmaciones recibidas de otros. Es decir, tratar de verificar conceptos antes de generalizarlos, mantener una postura dialéctica ante el pensar y el hacer.

Actitud investigadora. Pasar de la intención al acto, organizar actividades que permitan encaminarse en un objetivo, mediante el trabajo en equipo; buscar la consecución de un objetivo a través de la resolución de los problemas.

Apertura a los otros. Tener en cuenta a los otros, tanto en lo que se refiere al conocimiento -comunicación- como a la acción -cooperación-, lo cual se hará evidente a través de respetar las ideas del grupo, respetar las reglas de comunicación de un contexto. Esto implica que en la nueva pedagogía hay que crear escenarios de trabajo en equipo, en los cuales se desarrollen roles, se permita el diálogo, la contrastación y se facilite la expresión del estudiante con sus pares.

Toma de conciencia de la utilización del medio social y natural. Tener la intención de mantener la vida y respetarla.

En síntesis, se debe analizar la situación del docente colombiano, quien desarrolla poca investigación, lo cual limita su experiencia en metodologías susceptibles de introducirse en el aula. Si el docente no se preocupa por su actitud frente a la investigación y frente a sus propias actitudes de corte científico, difícilmente podrá motivar en sus estudiantes cualidades relacionadas con este tema. El desarrollo sólo teórico de los contenidos llevan a la monotonía; sin embargo, alternativas metodológicas que favorezcan el planteamiento de problemas, la resolución de los mismos, la confrontación de puntos de vista permiten la apropiación crítica del conocimiento. 


\section{Conclusiones}

En Colombia ha predominado la enseñanza con énfasis en los contenidos; sin embargo, es de vital importancia que se generen espacios en los que el estudiante conozca además de cómo se da origen a las teorías, reflexione sobre el proceso de construcción del conocimiento, lo cual lo llevará a indagar en la naturaleza de la ciencia, que a su vez, le permitirá una desmitificación de su concepto y forma de desarrollarse, acercándolo y familiarizándolo con ella.

El acercamiento de los estudiantes al trabajo científico es factible de realizarse dentro del aula y el docente debe ser un facilitador de dicho proceso. Para que efectivamente se pueda desarrollar un espíritu científico en los educandos, y hacer del aula un escenario en el que se motive "una actividad científica escolar", se debe regresar la mirada, primero, a los docentes, para que sean ellos quienes aborden la ciencia de una manera plural, como un constructo social, en el cual confluyen la historia, la cultura, la política, los sueños y las pasiones de seres humanos; por tanto es necesario conocer cómo piensa el maestro y qué referentes teóricos se suscriben a su práctica, para en consecuencia poder diseñar un plan de transformación (Porlán, 1993; Aduriz-Bravo, 2001).

Se aprehende mejor cuando el conocimiento toca las más profundas fibras, cuando lo que se quiere enseñar se acerca a lo que interesa y gusta; el momento es parte integral de un proceso, cuando se siente que se hace parte y juega un papel dentro de un colectivo, es por eso que, trabajar dentro de las Ciencias Naturales, reconociéndose como parte de ella, hará que el estudiante quiera a dichas ciencias, se interese en su aprendizaje al saber que son una construcción colectiva, en la que él de alguna manera, pueda participar en tal apasionante dinámica, de ahí la importancia de la historia como un referente para ser articulado a la enseñanza de las ciencias.

Los dos puntos sobre los cuales se basa la reflexión enunciada es hacer uso de la historia de las ciencias, para que se pueda construir una visión de la naturaleza de la misma, acorde con la construcción del espíritu científico, y además hacer dinámica la apropiación del conocimiento por parte del estudiante. 


\section{REFERENCIAS}

1. ADURIZ-BRAVO, Agustín y otros. (2001). Integración de la Epistemología en la formación del profesorado de Ciencias. Universidad Autónoma de Barcelona. Bellaterra.

2. (2003). Actualización en didáctica de las ciencias naturales y las matemáticas. Didácticas Magisterio. Bogotá.

3. (2005). Una introducción a la naturaleza de la ciencia. La epistemología en la enseñanza de las ciencias naturales. Fondo de Cultura Económica. Buenos Aires.

4. AGUIRRE, Juan Carlos y JARAMILLO, Luis Guillermo (2006). Consideraciones acerca de la investigación en el aula: más allá de estar a la moda. Universidad del Cauca. Colombia.

5. BACHELARD, Gastón (1988). La formación del espíritu científico. Siglo XXI. México.

6. (1997). La formación del espíritu científico. Siglo Veintiuno, 21 a edición. México.

7. (2003). La filosofía del no. Amorrortu, 4ª Reimpresión. Buenos Aires.

8. DE BONO, Eduard (1986). El pensamiento lateral. Paidós. Barcelona.

9. FEURASTEIN, Reueven (1993). Modificabilidad cognitiva y programa de enriquecimiento instrumental. Manual para el alumno y el docente. Instituto Superior Pío X. Madrid.

10. FREIRE, Paulo. (1996). Pedagogía de la liberación. América Latina. Bogotá.

11. (1996). Pedagogía del oprimido. América Latina. Bogotá.

12. GALLEGO BADILLO Rómulo y otros (2009). Una aproximación histórico epistemológica a las leyes fundamentales de la Química. Universidad Pedagógica Nacional. Bogotá, D. C. En: Revista Electrónica de Enseñanza de las Ciencias, Vol. 8 №. 1.

13. GIL, Daniel y Otros (1994). Formación del profesorado de las ciencias y la matemática, tendencias y experiencias innovadoras.

14. HERNANDEZ, Augusto (2004). Aproximación a un estado del arte de la enseñanza de las ciencias en Colombia. Universidad Nacional de Colombia.

15. HUGO, Diana y ADURIZ-BRAVO, Agustín (2003). Algunos elementos teóricos para la investigación del conocimiento profesional del profesorado de Ciencias Naturales acerca de la naturaleza de las ciencias. Universidad Autónoma de Barcelona.

16. IZQUIERDO, Mercé y ADURIZ-BRAVO, Agustín (2002). Relación de la didáctica de las Ciencias Naturales con otras disciplinas científicas. Universidad Autónoma de Barcelona.

17. IZQUIERDO, Mercedes (2002). Relaciones de la didáctica de las ciencias naturales con otras disciplinas científicas. Fondo de Cultura Económica. 
18. LOCARNINI, Gabriel Omar (2007). Enseñar Ciencias Naturales. ¿Para qué? Un ensayo de respuesta que argumente la importancia de la enseñanza de las Ciencias en el Nivel Educación General Básica. Ministerio de Educación del Ecuador.

19. LURIA, Alexander (1984). El cerebro en acción. Martínez Roca. Barcelona.

20. MAYER, Richard (1986) Pensamiento, resolución de problemas y cognición. Paidós. Barcelona.

21. MUÑOZ, Zulma y otros (1996). Los centros de interés como ejes motores de los programas guías de investigación. Universidad de Nariño. Especialización en Docencia de la Química.

22. PORLAN, Rafael (1993). Constructivismo y escuela. Hacia un modelo de enseñanza-aprendizaje basado en la investigación. Diada editorial. Sevilla.

23. RUIZ LIMÓN, Ramón. Historia y evolución del pensamiento científico. Biblioteca virtual de derecho.

24. STERNBERG, Robert y SPEAR-SWERLING, Louise (2000). Enseñar a pensar. Santillana. Madrid.

25. TOURAINE, Alain (2000). Crítica de la modernidad. Fondo de Cultura Económica. Bogotá.

26. VEGA, M. (1989). Introducción a la psicología cognitiva. Alianza editorial. Madrid. 